\title{
Addition of $\boldsymbol{n}$-Butyllithium to an Aldimine:
}

\section{On the Role of Chelation, Aggregation, and Cooperative Solvation}

\author{
Bo Qu and David B. Collum* \\ Department of Chemistry and Chemical Biology \\ Baker Laboratory, Cornell University \\ Ithaca, New York 14853-1301
}

\section{Supporting Information}

\section{${ }^{6}$ Li NMR Spectra and IR Kinetics Studies}

I. ${ }^{6} \mathrm{Li} \mathrm{NMR}$ spectra of $\left[{ }^{6} \mathrm{Li}\right] n$-BuLi containing $\mathrm{Et}_{2} \mathrm{O}$ and/or TMEDA.

II. Plot of $k_{\text {obsd }}$ Vs [TMEDA] for the 1,2-addition of $n$-BuLi to imine $\mathbf{1}$ in cumene cosolvent.

III. Table of data for plot in Section II.

IV. Plot of $k_{\text {obsd }}$ Vs [ $n$-BuLi] for the 1,2-addition of $n$-BuLi to imine $\mathbf{1}$ in $0.05 \mathrm{M}$ TMEDA and toluene cosolvent.

V. Table of data for plot in Section IV.

VI. Plot of $k_{\text {obsd }}$ vs [ $n$-BuLi] for the 1,2-addition of $n$-BuLi to imine $\mathbf{1}$ in 1.50 M TMEDA and cumene cosolvent.

VII. Table of data for plot in Section VI.

VIII. Plot of $k_{\text {obsd }}$ vs [ $\mathrm{Et}_{2} \mathrm{O}$ ] for the 1,2-addition of $n$-BuLi to imine $\mathbf{1}$ in TMEDA and toluene cosolvent.

IX. Table of data for plot in Section VIII.

X. Plot of $k_{\text {obsd }}$ Vs [TMEDA] for the 1,2-addition of $n$-BuLi to imine $\mathbf{1}$ in $\mathrm{Et}_{2} \mathrm{O}$ and toluene cosolvent.

XI. Table of data for plot in Section X. 
XII. Plot of $k_{\text {obsd }}$ vs [TMEDA] for the 1,2-addition of $n$-BuLi to imine $\mathbf{1}$ in toluene cosolvent with variable $\mathrm{Et}_{2} \mathrm{O}$ concentrations.

XIII. Table of data for plots in Section XII.

XIV. Plot of $k_{\text {obsd }}$ vs [Et ${ }_{2} \mathrm{O}$ ] for the 1,2-addition of $n$-BuLi to imine $\mathbf{1}$ in toluene cosolvent with variable TMEDA concentrations.

XV. Table of data for plots in Section XIV.

XVI. Plot of $k_{\text {obsd }}$ vs [n-BuLi] for the 1,2-addition of $n$-BuLi to imine $\mathbf{1}$ in $\mathrm{Et}_{2} \mathrm{O}$ and 0.10 M TMEDA in toluene cosolvent.

XVII. Table of data for plot in Section XVI.

XVIII. Plot of $k_{\text {obsd }}$ vs [ $n$-BuLi] for the 1,2-addition of $n$-BuLi to imine $\mathbf{1}$ in $\mathrm{Et}_{2} \mathrm{O}$ and 0.90 M TMEDA in toluene cosolvent.

XIX. Table of data for plot in Section XVIII.

\section{Experimental Section}

I. General procedures.

II. Preparation of imine $\mathbf{1 .}$ 

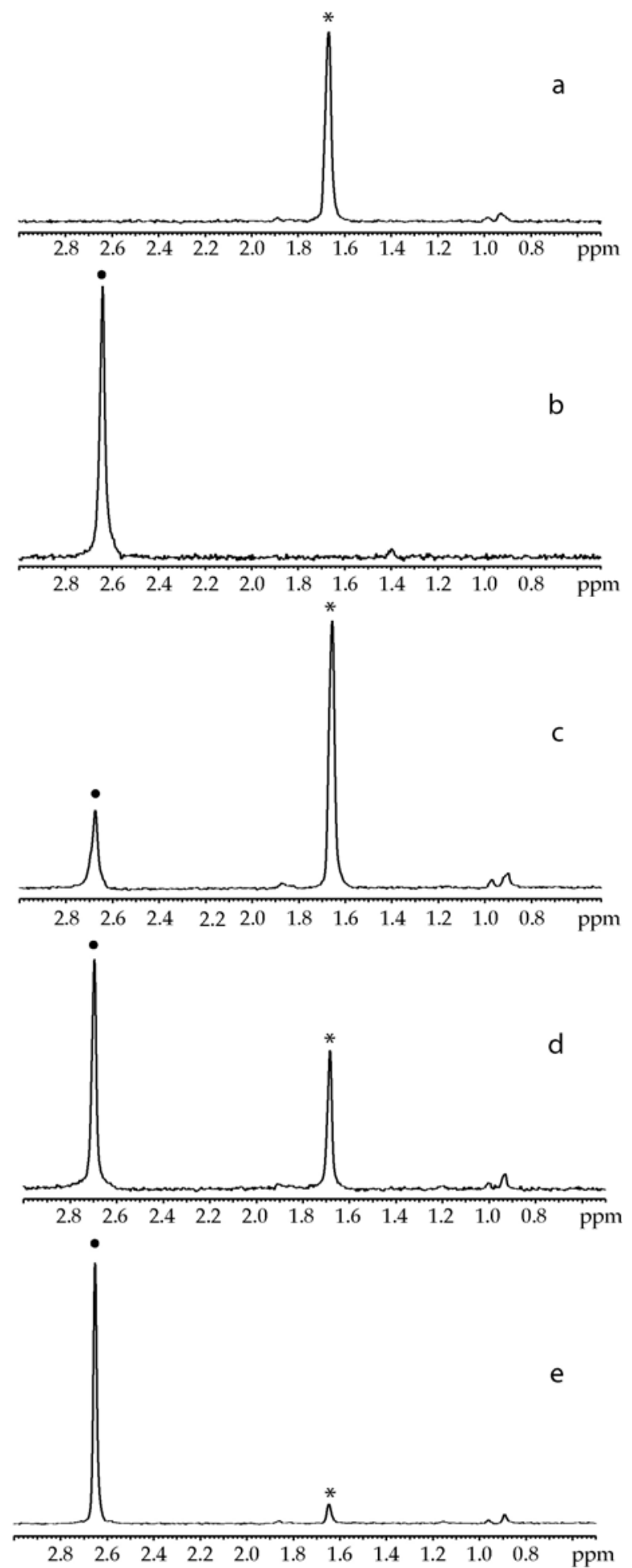

I. ${ }^{6} \mathrm{Li}$ NMR spectra of $0.10 \mathrm{M}\left[{ }^{6} \mathrm{Li}\right] n-\mathrm{BuLi}$ at $-110{ }^{\circ} \mathrm{C}$ containing: a) $5.0 \mathrm{M} \mathrm{Et}_{2} \mathrm{O}$; b) $1.0 \mathrm{M}$ TMEDA; c) $5.0 \mathrm{M} \mathrm{Et}_{2} \mathrm{O}$ and $0.02 \mathrm{M}$ TMEDA; d) $5.0 \mathrm{M} \mathrm{Et}_{2} \mathrm{O}$ and $0.05 \mathrm{M}$ TMEDA; e) 5.0 $\mathrm{M} \mathrm{Et}_{2} \mathrm{O}$ and $0.10 \mathrm{M}$ TMEDA. $\left(*(n-\mathrm{BuLi})_{4}\left(\mathrm{Et}_{2} \mathrm{O}\right)_{4}, \bullet(n-\mathrm{BuLi})_{2}(\mathrm{TMEDA})_{2}\right)$ 


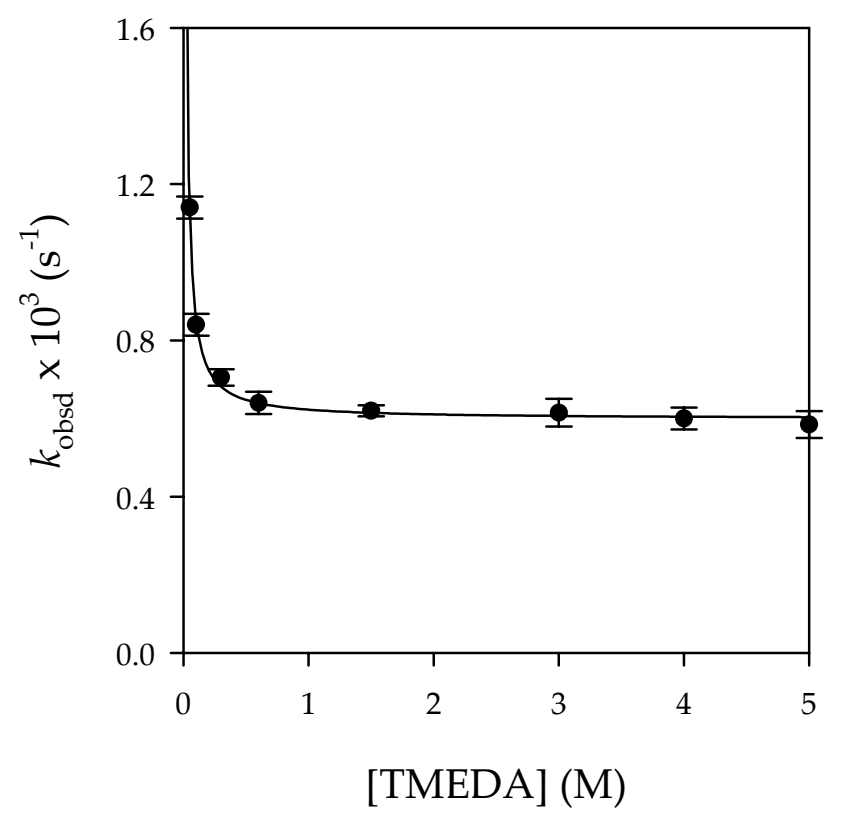

II. Plot of $k_{\text {obsd }}$ vs [TMEDA] for the 1,2-addition of $n-\operatorname{BuLi}(0.10 \mathrm{M})$ to imine $\mathbf{1}$ (0.007 M) in cumene ${ }^{*}$ cosolvent at $-55^{\circ} \mathrm{C}$. The curve depicts an unweighted least-squares fit to $k_{\mathrm{obsd}}=a[\text { TMEDA }]^{n}+b\left(a=3 \pm 1 \times 10^{-5}, n=-1.0 \pm 0.1, b=5.9 \pm 0.1 \times 10^{-4}\right)$.

* Cumene cosolvent was used instead of toluene at elevated temperature to prevent metallation of toluene by $n$-Buli in the presence of TMEDA.

III. Table of data for plot in Section II.

$\begin{array}{llll}\text { [TMEDA] (M) } & k_{\text {obsd }} 1\left(\mathrm{~s}^{-1}\right) & k_{\text {obsd }} 2\left(\mathrm{~s}^{-1}\right) & k_{\text {obsd }}(\mathrm{av})\left(\mathrm{s}^{-1}\right) \\ 0.05 & 0.00116 \pm 8 \mathrm{E}-5 & 0.00112 \pm 8 \mathrm{E}-5 & 0.00114 \pm 3 \mathrm{E}-5 \\ 0.10 & 0.00080 \pm 2 \mathrm{E}-5 & 0.00086 \pm 4 \mathrm{E}-5 & 0.00083 \pm 4 \mathrm{E}-5 \\ 0.30 & 0.00077 \pm 2 \mathrm{E}-5 & 0.00063 \pm 2 \mathrm{E}-5 & 0.0007 \pm 1 \mathrm{E}-4 \\ 0.60 & 0.00072 \pm 3 \mathrm{E}-5 & 0.00058 \pm 2 \mathrm{E}-5 & 0.00065 \pm 9 \mathrm{E}-5 \\ 1.50 & 0.00056 \pm 1 \mathrm{E}-5 & 0.00065 \pm 2 \mathrm{E}-5 & 0.00061 \pm 6 \mathrm{E}-5 \\ 3.0 & 0.00066 \pm 5 \mathrm{E}-5 & 0.00055 \pm 9 \mathrm{E}-5 & 0.00061 \pm 6 \mathrm{E}-5 \\ 4.0 & 0.00056 \pm 1 \mathrm{E}-5 & 0.00064 \pm 1 \mathrm{E}-5 & 0.00060 \pm 6 \mathrm{E}-5 \\ 5.0 & 0.000609 \pm 9 \mathrm{E}-6 & 0.00052 \pm 1 \mathrm{E}-5 & 0.00056 \pm 6 \mathrm{E}-5\end{array}$




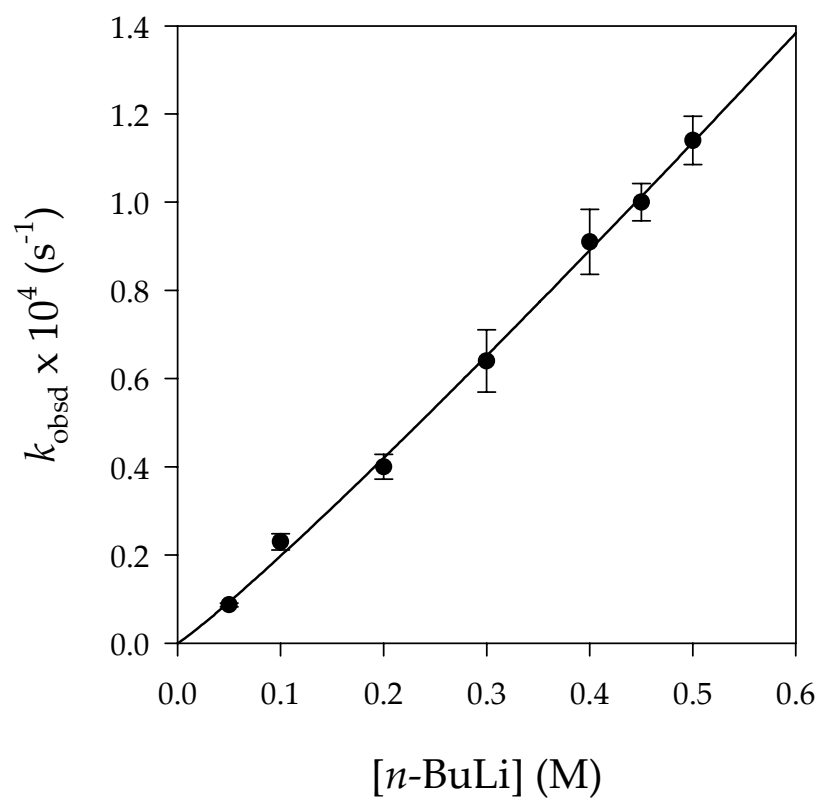

IV. Plot of $k_{\text {obsd }}$ vs [ $n$-BuLi] for the 1,2-addition of $n$-BuLi to imine $\mathbf{1}(0.007 \mathrm{M})$ in 0.05 $\mathrm{M}$ TMEDA and toluene cosolvent at $-78^{\circ} \mathrm{C}$. The curve depicts an unweighted leastsquares fit to $k_{\mathrm{obsd}}=a[n-\mathrm{BuLi}]^{b}\left(a=2.4 \pm 0.1 \times 10^{-4}, b=1.09 \pm 0.03\right)$. Alternatively, fit to $k_{\mathrm{obsd}}=a[n-\mathrm{BuLi}]^{b}+k^{\prime}[n-\mathrm{BuLi}]^{1 / 2}\left(a=2.4 \pm 0.1 \times 10^{-4}, b=1.16 \pm 0.04\right)$, where $k^{\prime}=1.14$ $\mathrm{x} 10^{-5}$, the $b$ term from Section XIII with no added $\mathrm{Et}_{2} \mathrm{O}$.

V. Table of data for plot in Section IV.
$[n-\mathrm{BuLi}](\mathrm{M}) \quad k_{\mathrm{obsd}} 1\left(\mathrm{~s}^{-1}\right)$
$k_{\mathrm{obsd}} 2\left(\mathrm{~s}^{-1}\right)$
$k_{\text {obsd }} 3\left(\mathrm{~s}^{-1}\right)$
$k_{\text {obsd }}\left(\right.$ av) $\left(\mathrm{s}^{-1}\right)$
0.05
$0.000009 \pm 4 \mathrm{E}-6$
$0.0000084 \pm 8 \mathrm{E}-7$
$0.0000087 \pm 4 \mathrm{E}-7$
0.10
$0.000024 \pm 1 \mathrm{E}-6$
$0.0000214 \pm 6 \mathrm{E}-7$
$0.000023 \pm 2 \mathrm{E}-6$
0.20
$0.000038 \pm 2 \mathrm{E}-6$
$0.000042 \pm 2 \mathrm{E}-6$
$0.000040 \pm 3 \mathrm{E}-6$
0.30
$0.000059 \pm 3 \mathrm{E}-6$
$0.000069 \pm 1 \mathrm{E}-6$
$0.000064 \pm 7 \mathrm{E}-6$
0.40
$0.000085 \pm 3 \mathrm{E}-6$
$0.000088 \pm 3 \mathrm{E}-6$
$0.000099 \pm 1 \mathrm{E}-6$
$0.000091 \pm 7 \mathrm{E}-6$
0.45
$0.000097 \pm 3 \mathrm{E}-6$
$0.000103 \pm 5 \mathrm{E}-6$
$0.000100 \pm 4 \mathrm{E}-6$
0.50
$0.000101 \pm 2 \mathrm{E}-6$
$0.000120 \pm 3 \mathrm{E}-6$
$0.000114 \pm 8 \mathrm{E}-6$ 


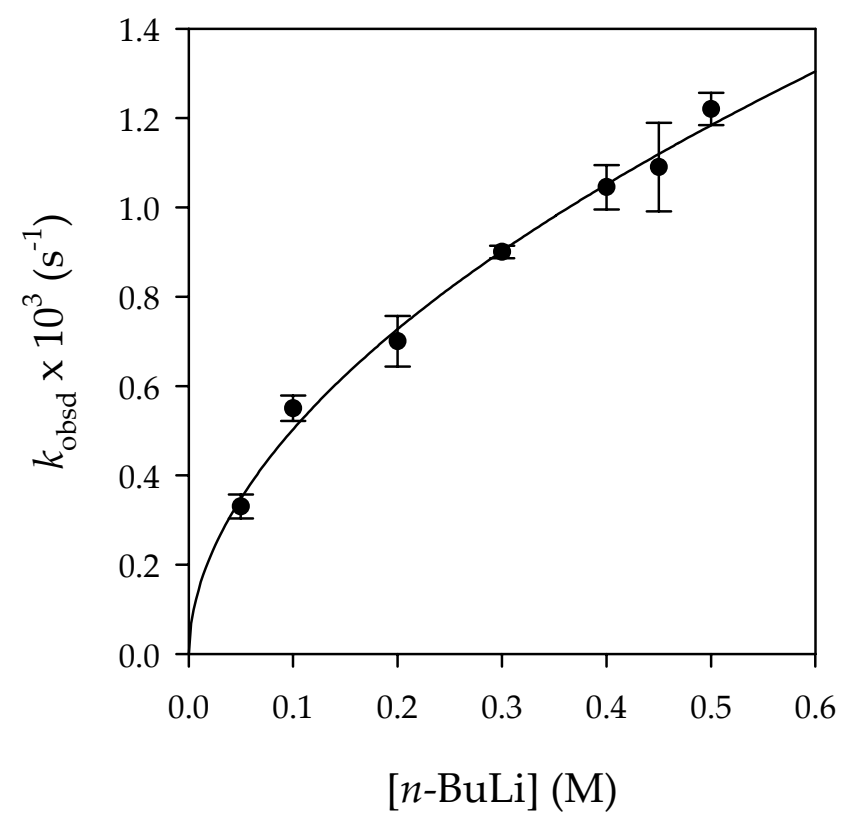

VI. Plot of $k_{\text {obsd }}$ Vs [ $n$-BuLi] for the 1,2-addition of $n$-BuLi to imine $1(0.007 \mathrm{M})$ in 1.50 $\mathrm{M}$ TMEDA and cumene cosolvent at $-55^{\circ} \mathrm{C}$. The curve depicts an unweighted leastsquares fit to $k_{\mathrm{obsd}}=a[n-\mathrm{BuLi}]^{b}\left(a=1.71 \pm 0.06 \times 10^{-3}, b=0.53 \pm 0.03\right)$.

VII. Table of data for plot in Section VI.

$\begin{array}{lllll}{[n-\mathrm{BuLi}](\mathrm{M})} & k_{\mathrm{obsd}} 1\left(\mathrm{~s}^{-1}\right) & k_{\mathrm{obsd}} 2\left(\mathrm{~s}^{-1}\right) & k_{\mathrm{obsd}} 3\left(\mathrm{~s}^{-1}\right) & k_{\mathrm{obsd}}(\mathrm{av})\left(\mathrm{s}^{-1}\right) \\ & & & \\ 0.05 & 0.000350 \pm 6 \mathrm{E}-6 & 0.000312 \pm 6 \mathrm{E}-6 & 0.00033 \pm 3 \mathrm{E}-5 \\ 0.10 & 0.00057 \pm 1 \mathrm{E}-5 & 0.00053 \pm 1 \mathrm{E}-5 & 0.00055 \pm 3 \mathrm{E}-5 \\ 0.20 & 0.00074 \pm 2 \mathrm{E}-5 & 0.00066 \pm 3 \mathrm{E}-5 & 0.00070 \pm 6 \mathrm{E}-5 \\ 0.30 & 0.00091 \pm 2 \mathrm{E}-5 & 0.00089 \pm 3 \mathrm{E}-5 & 0.0009 \pm 1 \mathrm{E}-5 \\ 0.40 & 0.00101 \pm 5 \mathrm{E}-5 & 0.00108 \pm 5 \mathrm{E}-5 & 0.00105 \pm 5 \mathrm{E}-5 \\ 0.45 & 0.00102 \pm 6 \mathrm{E}-5 & 0.00116 \pm 5 \mathrm{E}-5 & & 0.0011 \pm 1 \mathrm{E}-4 \\ 0.50 & 0.00118 \pm 7 \mathrm{E}-5 & 0.00125 \pm 7 \mathrm{E}-5 & 0.00123 \pm 6 \mathrm{E}-5 & 0.00122 \pm 4 \mathrm{E}-5\end{array}$




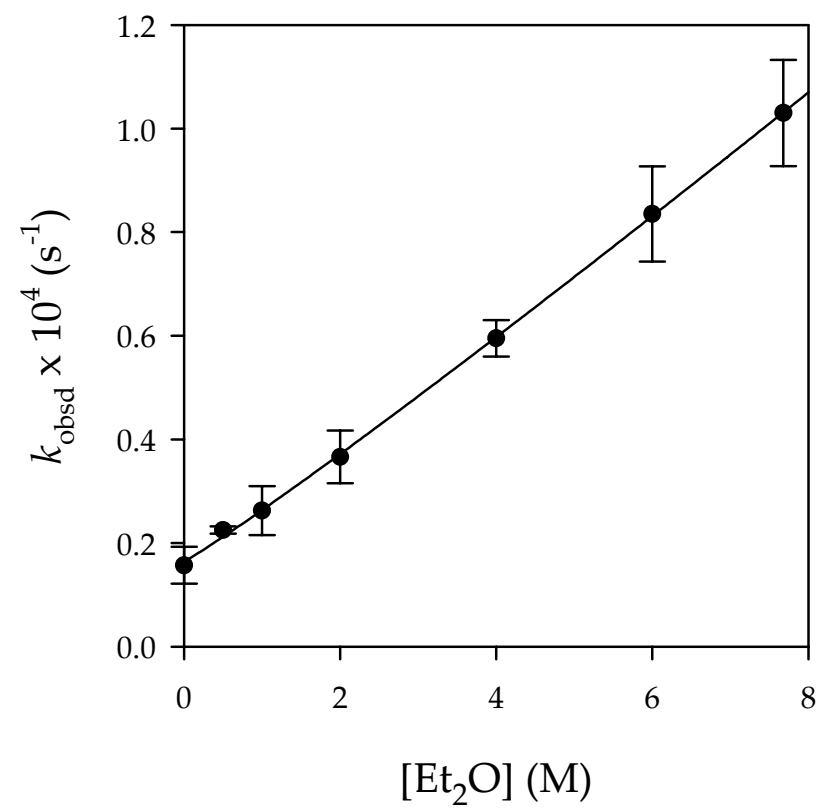

VIII. Plot of $k_{\text {obsd }}$ Vs [Et ${ }_{2} \mathrm{O}$ ] for the 1,2-addition of $n-\operatorname{BuLi}(0.10 \mathrm{M})$ to imine $1(0.007 \mathrm{M})$ in $0.90 \mathrm{M}$ TMEDA and toluene cosolvent at $-78^{\circ} \mathrm{C}$. The curve depicts an unweighted least-squares fit to $k_{\mathrm{obsd}}=a\left[\mathrm{Et}_{2} \mathrm{O}\right]^{n}+b\left(a=1.0 \pm 0.1 \times 10^{-5}, n=1.06 \pm 0.03, b=1.6 \pm\right.$ $\left.0.1 \times 10^{-5}\right)$.

IX. Table of data for plot in Section VIII.
$\left[\mathrm{Et}_{2} \mathrm{O}\right](\mathrm{M})$
$k_{\text {obsd }} 1\left(\mathrm{~s}^{-1}\right)$
$k_{\mathrm{obsd}} 2\left(\mathrm{~s}^{-1}\right)$
$k_{\text {obsd }}(\mathrm{av})\left(\mathrm{s}^{-1}\right)$
0.0
$0.0000132 \pm 8 \mathrm{E}-7$
$0.0000182 \pm 9 \mathrm{E}-7$
$0.000016 \pm 4 \mathrm{E}-6$
0.5
$0.000023 \pm 1 \mathrm{E}-6$
$0.000022 \pm 2 \mathrm{E}-5$
$0.0000225 \pm 7 \mathrm{E}-7$
1.0
$0.0000229 \pm 6 \mathrm{E}-7$
$0.0000296 \pm 6 \mathrm{E}-7$
$0.000026 \pm 5 \mathrm{E}-6$
2.0
$0.000033 \pm 3 \mathrm{E}-6$
$0.0000402 \pm 8 \mathrm{E}-7$
$0.0000366 \pm 5 \mathrm{E}-6$
4.0
$0.000062 \pm 1 \mathrm{E}-6$
$0.000057 \pm 2 \mathrm{E}-6$
$0.0000595 \pm 4 \mathrm{E}-6$
6.0
$0.000090 \pm 3 \mathrm{E}-6$
$0.000077 \pm 3 \mathrm{E}-6$
$0.000084 \pm 9 \mathrm{E}-6$
7.68
$0.0001105 \pm 3 \mathrm{E}-7 \quad 0.000096 \pm 2 \mathrm{E}-6$
$0.00010 \pm 1 \mathrm{E}-5$ 


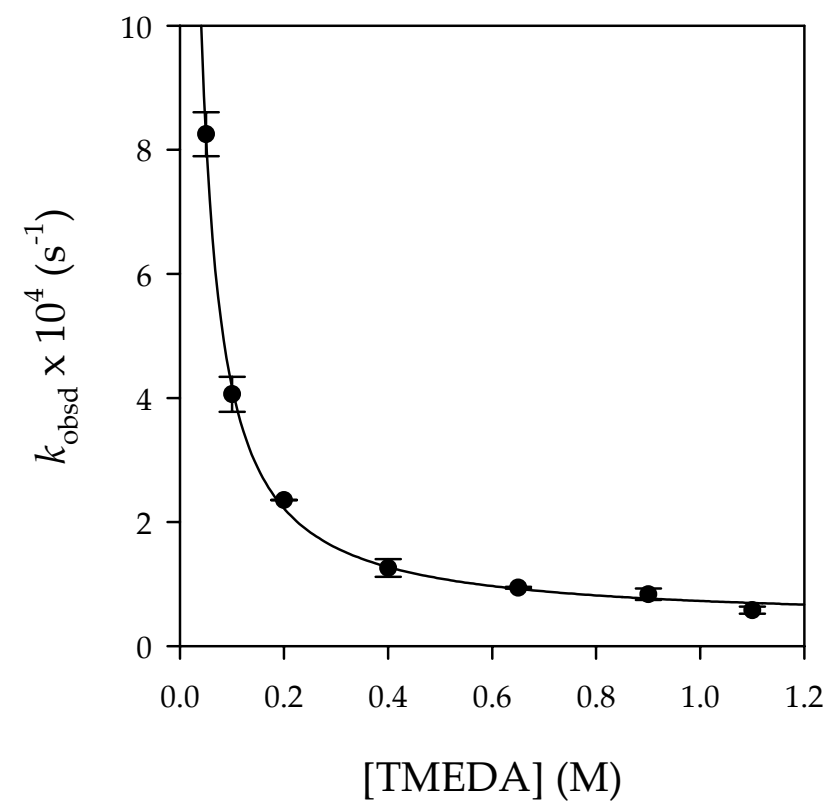

X. Plot of $k_{\text {obsd }}$ vs [TMEDA] for the 1,2-addition of $n-\operatorname{BuLi}(0.10 \mathrm{M})$ to imine $\mathbf{1}(0.007$ $\mathrm{M})$ in $6.0 \mathrm{M} \mathrm{Et}_{2} \mathrm{O}$ and toluene cosolvent at $-78{ }^{\circ} \mathrm{C}$. The curve depicts an unweighted least-squares fit to $k_{\mathrm{obsd}}=a[\mathrm{TMEDA}]^{n}+b\left(a=3.4 \pm 0.6 \times 10^{-5}, n=-1.05 \pm 0.05, b=3.9\right.$ $\left.\pm 1.1 \times 10^{-5}\right)$.

XI. Table of data for plot in Section X.
[TMEDA] (M) $k_{\text {obsd }} 1\left(\mathrm{~s}^{-1}\right)$
$k_{\text {obsd }} 2\left(\mathrm{~s}^{-1}\right)$
$k_{\text {obsd }}$ (av) $\left(\mathrm{s}^{-1}\right)$
0.05
$0.00080 \pm 2 \mathrm{E}-5$
$0.00085 \pm 2 \mathrm{E}-5$
$0.00083 \pm 4 \mathrm{E}-5$
0.10
$0.000426 \pm 7 \mathrm{E}-6$
$0.000386 \pm 6 \mathrm{E}-6$
$0.000406 \pm 3 \mathrm{E}-5$
0.20
$0.000241 \pm 3 \mathrm{E}-6$
$0.000230 \pm 5 \mathrm{E}-6$
$0.000236 \pm 8 \mathrm{E}-6$
0.40
$0.000136 \pm 2 \mathrm{E}-6$
$0.000116 \pm 7 \mathrm{E}-6$
$0.00013 \pm 1 \mathrm{E}-5$
0.65
$0.000095 \pm 1 \mathrm{E}-6$
$0.000093 \pm 3 \mathrm{E}-6$
$0.000094 \pm 1 \mathrm{E}-6$
0.90
$0.000094 \pm 3 \mathrm{E}-6 \quad 0.000074 \pm 1 \mathrm{E}-6$
$0.000084 \pm 9 \mathrm{E}-6$
1.10
$0.0000538 \pm 4 \mathrm{E}-7 \quad 0.000062 \pm 8 \mathrm{E}-6$
$0.0000579 \pm 6 \mathrm{E}-6$ 


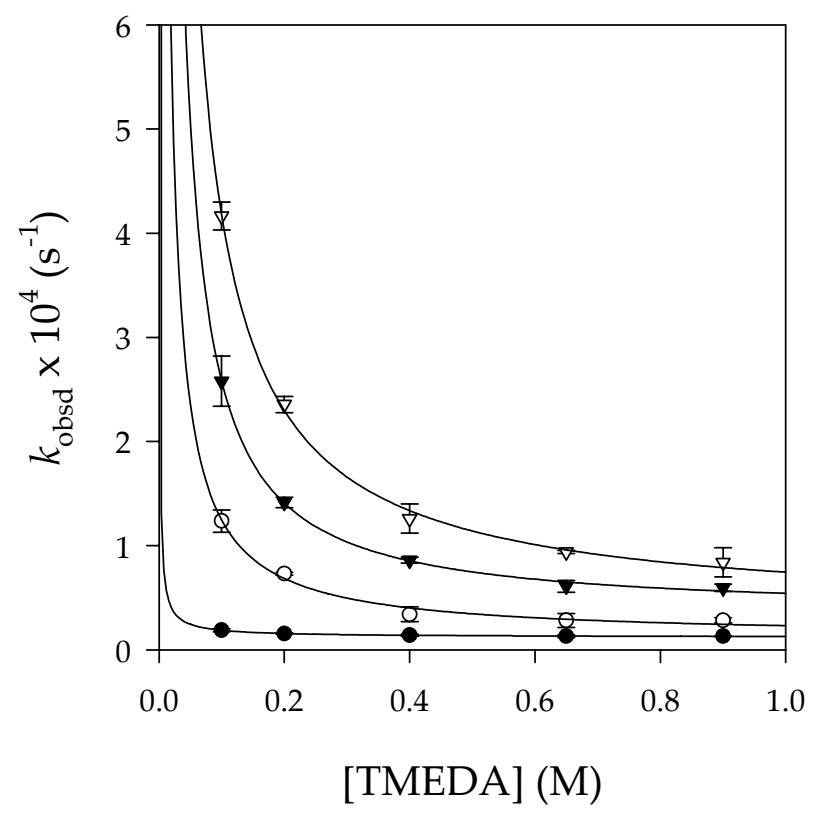

XII. Plot of $k_{\text {obsd }}$ vs [TMEDA] for the 1,2-addition of $n-\operatorname{BuLi}(0.10 \mathrm{M})$ to imine $\mathbf{1}(0.007$ $\mathrm{M})$ in toluene cosolvent at $-78{ }^{\circ} \mathrm{C}$ with variable $\mathrm{Et}_{2} \mathrm{O}$ concentrations. $\nabla 6.0 \mathrm{M} \mathrm{Et}_{2} \mathrm{O}$, 4.0 $\mathrm{M} \mathrm{Et}_{2} \mathrm{O}, \circ 2.0 \mathrm{M} \mathrm{Et}_{2} \mathrm{O}, \bullet 0.0 \mathrm{M} \mathrm{Et}_{2} \mathrm{O}$. The curves depict unweighted least-squares fits to $k_{\mathrm{obsd}}=a[\text { TMEDA }]^{n}+b$.

XIII. Table of data for plot in Section XII.

$6.0 \mathrm{M} \mathrm{Et}_{2} \mathrm{O}$

$k_{\mathrm{obsd}}=a[\text { TMEDA }]^{n}+b\left(a=4.1 \pm 1.2 \times 10^{-5}, b=-1.0 \pm 0.1, b=3.3 \pm 1.8 \times 10^{-5}\right)$.

[TMEDA] (M) $k_{\text {obsd }} 1\left(\mathrm{~s}^{-1}\right) \quad k_{\text {obsd }} 2\left(\mathrm{~s}^{-1}\right) \quad k_{\text {obsd }}($ av $)\left(\mathrm{s}^{-1}\right)$

$0.10 \quad 0.000426 \pm 7 \mathrm{E}-6 \quad 0.000386 \pm 7 \mathrm{E}-6 \quad 0.00041 \pm 3 \mathrm{E}-5$

$0.20 \quad 0.000228 \pm 5 \mathrm{E}-6 \quad 0.000239 \pm 3 \mathrm{E}-6 \quad 0.000236 \pm 8 \mathrm{E}-6$

$0.40 \quad 0.000116 \pm 7 \mathrm{E}-6 \quad 0.000136 \pm 2 \mathrm{E}-6 \quad 0.00013 \pm 1 \mathrm{E}-5$

$0.65 \quad 0.000095 \pm 1 \mathrm{E}-6 \quad 0.000093 \pm 3 \mathrm{E}-6 \quad 0.000094 \pm 1 \mathrm{E}-6$

$0.90 \quad 0.000094 \pm 1 \mathrm{E}-6 \quad 0.000074 \pm 3 \mathrm{E}-6 \quad 0.000084 \pm 9 \mathrm{E}-6$ 


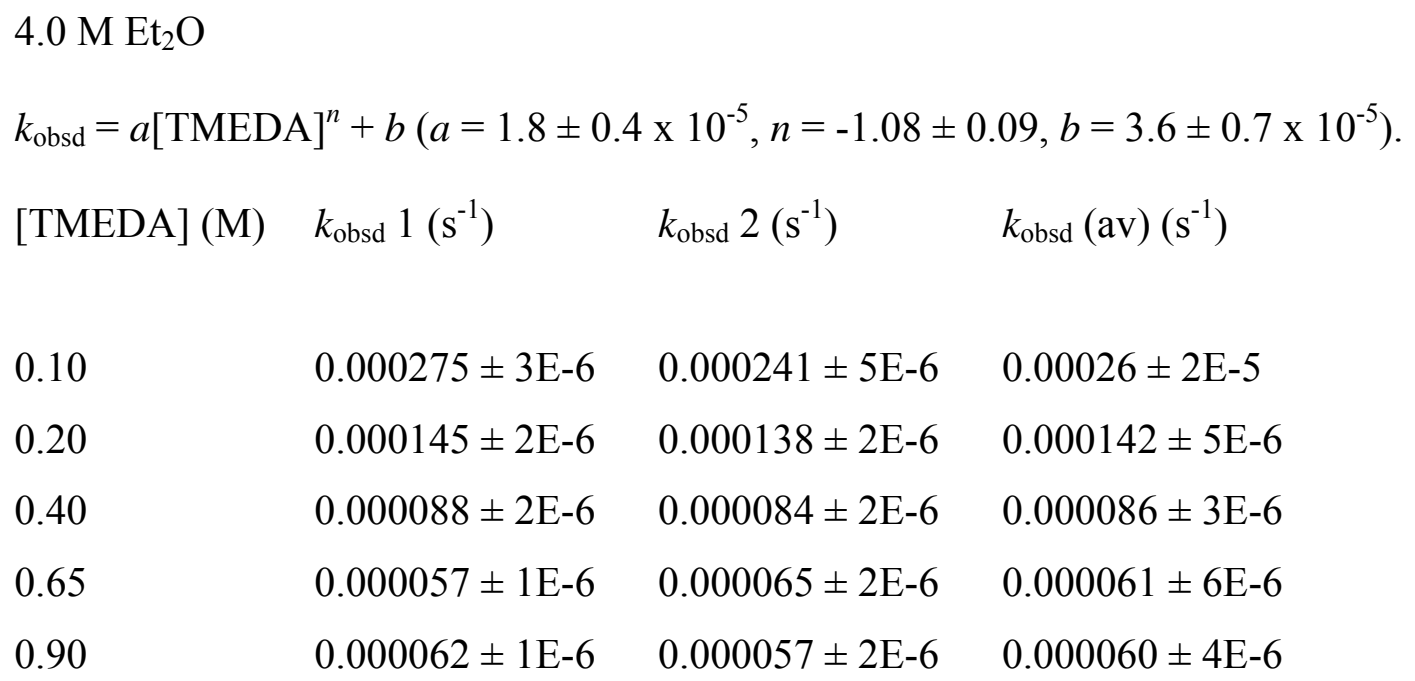

\section{$2.0 \mathrm{M} \mathrm{Et}_{2} \mathrm{O}$}

\begin{tabular}{|c|c|c|c|c|}
\hline [TMEDA] (M) & $k_{\text {obsd }} 1\left(\mathrm{~s}^{-1}\right)$ & $k_{\text {obsd }} 2\left(\mathrm{~s}^{-1}\right)$ & $k_{\text {obsd }} 3\left(\mathrm{~s}^{-1}\right)$ & $k_{\text {obsd }}(\mathrm{av})\left(\mathrm{s}^{-1}\right)$ \\
\hline 0.10 & $0.000112 \pm 1 \mathrm{E}-6$ & $0.000133 \pm 2 \mathrm{E}-6$ & $0.000126 \pm 5 \mathrm{E}-6$ & $0.00012 \pm 1 \mathrm{E}-5$ \\
\hline 0.20 & $0.000074 \pm 1 \mathrm{E}-6$ & $0.000076 \pm 2 \mathrm{E}-6$ & & $0.000073 \pm 1 \mathrm{E}-6$ \\
\hline 0.40 & $0.000029 \pm 1 \mathrm{E}-6$ & $0.000039 \pm 2 \mathrm{E}-6$ & & $0.000034 \pm 7 \mathrm{E}-6$ \\
\hline 0.65 & $0.0000235 \pm 5 \mathrm{E}-7$ & $0.0000329 \pm 3 \mathrm{E}-7$ & & $0.000028 \pm 7 \mathrm{E}-6$ \\
\hline 0.90 & $0.000030 \pm 3 \mathrm{E}-6$ & $0.0000265 \pm 2 \mathrm{E}-7$ & & $0.000028 \pm 2 \mathrm{E}-6$ \\
\hline
\end{tabular}

$0.0 \mathrm{M} \mathrm{Et}_{2} \mathrm{O}$

$k_{\mathrm{obsd}}=a[\text { TMEDA }]^{n}+b\left(a=1.4 \pm 0.7 \times 10^{-6}, n=-0.7 \pm 0.2, b=1.1 \pm 0.1 \times 10^{-5}\right)$.

[TMEDA] (M) $k_{\text {obsd }} 1\left(\mathrm{~s}^{-1}\right) \quad k_{\text {obsd }} 2\left(\mathrm{~s}^{-1}\right) \quad k_{\text {obsd }}($ av $)\left(\mathrm{s}^{-1}\right)$

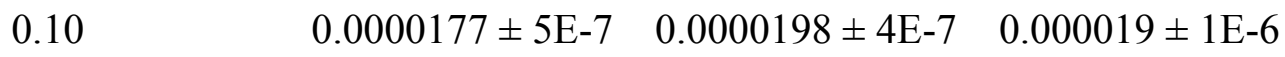

$0.20 \quad 0.0000152 \pm 5 \mathrm{E}-7 \quad 0.000016 \pm 4 \mathrm{E}-6 \quad 0.0000156 \pm 6 \mathrm{E}-7$

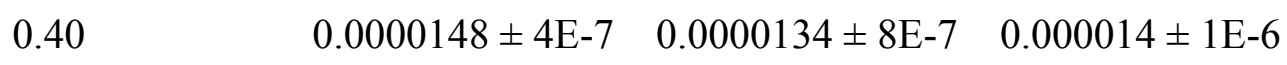

$0.65 \quad 0.0000140 \pm 2 \mathrm{E}-7 \quad 0.0000121 \pm 2 \mathrm{E}-7 \quad 0.000013 \pm 1 \mathrm{E}-6$

$0.90 \quad 0.0000132 \pm 8 \mathrm{E}-7 \quad 0.0000182 \pm 9 \mathrm{E}-7 \quad 0.000016 \pm 4 \mathrm{E}-6$ 


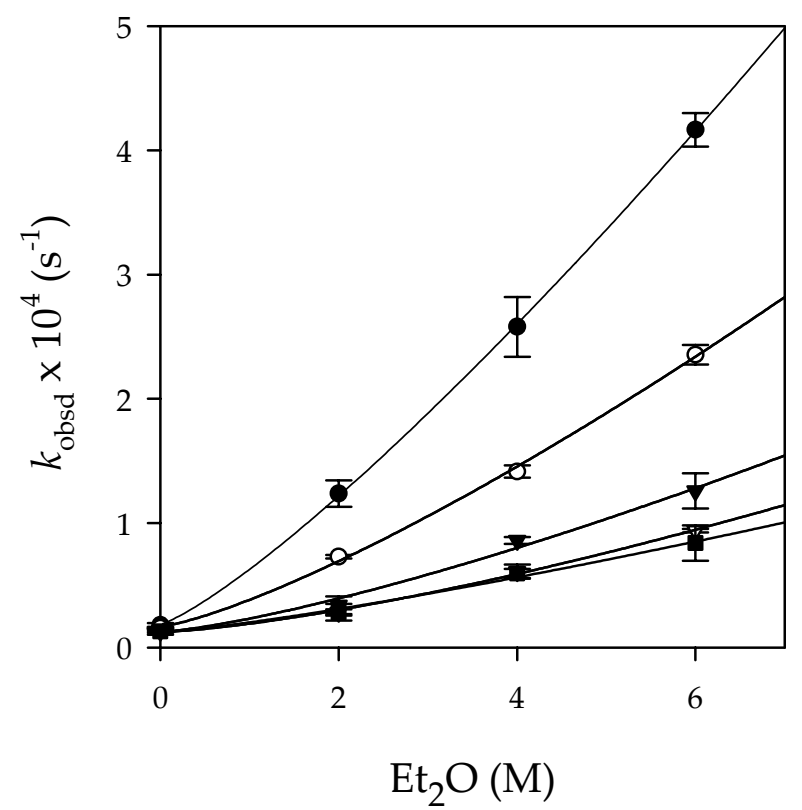

XIV. Plot of $k_{\text {obsd }}$ Vs [ $\mathrm{Et}_{2} \mathrm{O}$ ] for the 1,2-addition of $n-\mathrm{BuLi}(0.10 \mathrm{M})$ to imine $1(0.007 \mathrm{M})$ in toluene cosolvent at $-78{ }^{\circ} \mathrm{C}$ with TMEDA. $\bullet 0.10 \mathrm{M}$ TMEDA, $0.20 \mathrm{M}$ TMEDA, $\boldsymbol{\nabla}$ $0.40 \mathrm{M}$ TMEDA, $\nabla 0.65 \mathrm{M}$ TMEDA, $\square .90 \mathrm{M}$ TMEDA. The curves depict unweighted least-squares fits to $k_{\mathrm{obsd}}=a\left[\mathrm{Et}_{2} \mathrm{O}\right]^{n}+b$.

XV. Table of data for plot in Section XIV.

0.10 M TMEDA

$k_{\mathrm{obsd}}=a\left[\mathrm{Et}_{2} \mathrm{O}\right]^{n}+b\left(a=4.64 \pm 0.01 \times 10^{-5}, n=1.18 \pm 0.01, b=1.81 \pm 0.01 \times 10^{-5}\right)$.

$\left[\mathrm{Et}_{2} \mathrm{O}\right](\mathrm{M}) \quad k_{\text {obsd }} 1\left(\mathrm{~s}^{-1}\right) \quad k_{\text {obsd }} 2\left(\mathrm{~s}^{-1}\right) \quad k_{\text {obsd }} 3\left(\mathrm{~s}^{-1}\right) \quad k_{\text {obsd }}(\mathrm{av})\left(\mathrm{s}^{-1}\right)$

$0.0 \quad 0.0000177 \pm 5 \mathrm{E}-7 \quad 0.0000198 \pm 4 \mathrm{E}-7 \quad 0.0000126 \pm 5 \mathrm{E}-6 \quad 0.000019 \pm 1 \mathrm{E}-6$

$2.0 \quad 0.000112 \pm 1 \mathrm{E}-6 \quad 0.000133 \pm 2 \mathrm{E}-6 \quad 0.00012 \pm 1 \mathrm{E}-5$

$4.0 \quad 0.000275 \pm 3 \mathrm{E}-6 \quad 0.000241 \pm 5 \mathrm{E}-6 \quad 0.00026 \pm 2 \mathrm{E}-5$

$6.0 \quad 0.000386 \pm 7 \mathrm{E}-6 \quad 0.000426 \pm 7 \mathrm{E}-6 \quad 0.00041 \pm 3 \mathrm{E}-5$

\subsection{TMEDA}

$k_{\mathrm{obsd}}=a\left[\mathrm{Et}_{2} \mathrm{O}\right]^{n}+b\left(a=2.2 \pm 0.4 \times 10^{-5}, n=1.2 \pm 0.1, b=1.7 \pm 0.6 \times 10^{-5}\right)$.

$\left[\mathrm{Et}_{2} \mathrm{O}\right](\mathrm{M}) \quad k_{\text {obsd }} 1\left(\mathrm{~s}^{-1}\right) \quad k_{\text {obsd }} 2\left(\mathrm{~s}^{-1}\right) \quad k_{\text {obsd }}(\mathrm{av})\left(\mathrm{s}^{-1}\right)$ 


$\begin{array}{llll}0.0 & 0.0000152 \pm 5 \mathrm{E}-7 & 0.000016 \pm 4 \mathrm{E}-6 & 0.0000156 \pm 6 \mathrm{E}-7 \\ 2.0 & 0.000074 \pm 1 \mathrm{E}-6 & 0.000076 \pm 2 \mathrm{E}-6 & 0.000730 \pm 1 \mathrm{E}-6 \\ 4.0 & 0.000145 \pm 2 \mathrm{E}-6 & 0.000138 \pm 2 \mathrm{E}-6 & 0.000142 \pm 5 \mathrm{E}-6 \\ 6.0 & 0.000228 \pm 5 \mathrm{E}-6 & 0.000239 \pm 3 \mathrm{E}-6 & 0.000236 \pm 1 \mathrm{E}-6\end{array}$

0.4 M TMEDA

$k_{\mathrm{obsd}}=a\left[\mathrm{Et}_{2} \mathrm{O}\right]^{n}+b\left(a=1.1 \pm 0.6 \times 10^{-5}, n=1.3 \pm 0.2, b=1.2 \pm 0.8 \times 10^{-5}\right)$.

$\left[\mathrm{Et}_{2} \mathrm{O}\right](\mathrm{M}) \quad k_{\text {obsd }} 1\left(\mathrm{~s}^{-1}\right) \quad k_{\text {obsd }} 2\left(\mathrm{~s}^{-1}\right) \quad k_{\text {obsd }}(\mathrm{av})\left(\mathrm{s}^{-1}\right)$

$0.0 \quad 0.0000148 \pm 4 \mathrm{E}-7 \quad 0.0000134 \pm 8 \mathrm{E}-7 \quad 0.000014 \pm 1 \mathrm{E}-6$

$2.0 \quad 0.000029 \pm 1 \mathrm{E}-6 \quad 0.000039 \pm 2 \mathrm{E}-6 \quad 0.000034 \pm 7 \mathrm{E}-6$

$\begin{array}{llll}4.0 & 0.000088 \pm 2 \mathrm{E}-6 & 0.000084 \pm 2 \mathrm{E}-6 & 0.000086 \pm 3 \mathrm{E}-6\end{array}$

$6.0 \quad 0.000116 \pm 7 \mathrm{E}-6 \quad 0.000136 \pm 2 \mathrm{E}-6 \quad 0.00013 \pm 1 \mathrm{E}-5$

0.65 M TMEDA

$k_{\mathrm{obsd}}=a\left[\mathrm{Et}_{2} \mathrm{O}\right]^{n}+b\left(a=6.7 \pm 1.9 \times 10^{-6}, n=1.3 \pm 0.2, b=1.2 \pm 0.3 \times 10^{-5}\right)$.

$\left[\mathrm{Et}_{2} \mathrm{O}\right](\mathrm{M}) \quad k_{\text {obsd }} 1\left(\mathrm{~s}^{-1}\right) \quad k_{\text {obsd }} 2\left(\mathrm{~s}^{-1}\right) \quad k_{\text {obsd }}(\mathrm{av})\left(\mathrm{s}^{-1}\right)$

$\begin{array}{llll}0.0 & 0.0000140 \pm 2 \mathrm{E}-7 & 0.0000121 \pm 2 \mathrm{E}-7 & 0.000013 \pm 1 \mathrm{E}-6\end{array}$

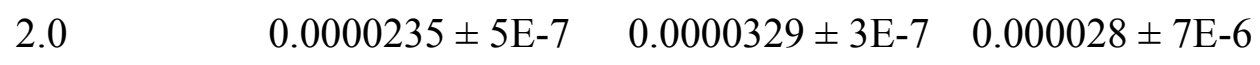

$\begin{array}{llll}4.0 & 0.000057 \pm 1 \mathrm{E}-6 & 0.000065 \pm 2 \mathrm{E}-6 & 0.000061 \pm 6 \mathrm{E}-6\end{array}$

$6.0 \quad 0.000093 \pm 3 \mathrm{E}-6 \quad 0.000095 \pm 1 \mathrm{E}-6 \quad 0.000094 \pm 1 \mathrm{E}-6$

0.90 M TMEDA

$k_{\mathrm{obsd}}=a\left[\mathrm{Et}_{2} \mathrm{O}\right]^{n}+b\left(a=7.9 \pm 3.4 \times 10^{-6}, n=1.2 \pm 0.2, b=1.2 \pm 0.4 \times 10^{-5}\right)$.

$\left[\mathrm{Et}_{2} \mathrm{O}\right](\mathrm{M}) \quad k_{\text {obsd }} 1\left(\mathrm{~s}^{-1}\right) \quad k_{\text {obsd }} 2\left(\mathrm{~s}^{-1}\right) \quad k_{\text {obsd }}(\mathrm{av})\left(\mathrm{s}^{-1}\right)$

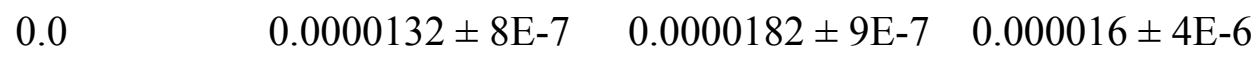

$2.0 \quad 0.000030 \pm 3 \mathrm{E}-6 \quad 0.0000265 \pm 2 \mathrm{E}-7 \quad 0.000028 \pm 2 \mathrm{E}-6$

$\begin{array}{llll}4.0 & 0.000057 \pm 2 \mathrm{E}-6 & 0.000062 \pm 1 \mathrm{E}-6 & 0.000060 \pm 4 \mathrm{E}-6\end{array}$

$6.0 \quad 0.000074 \pm 3 \mathrm{E}-6 \quad 0.000094 \pm 1 \mathrm{E}-6 \quad 0.000084 \pm 9 \mathrm{E}-6$ 


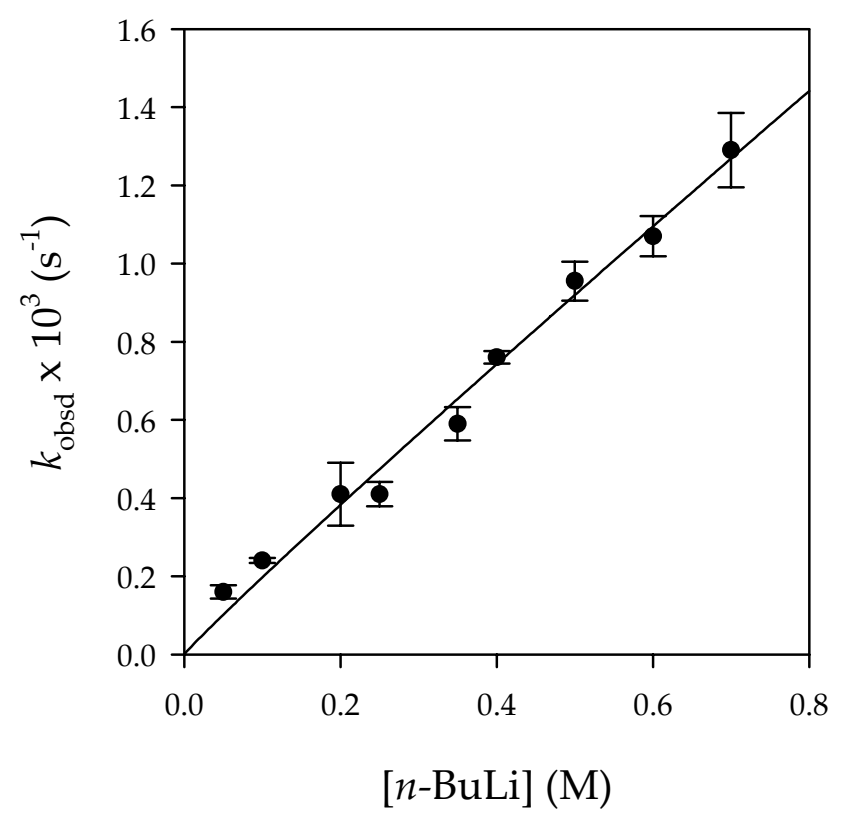

XVI. Plot of $k_{\text {obsd }}$ Vs [ $n$-BuLi] for the 1,2-addition of $n$-BuLi to imine $\mathbf{1}(0.007 \mathrm{M})$ in 4.0 $\mathrm{M} \mathrm{Et}_{2} \mathrm{O}$ and $0.10 \mathrm{M}$ TMEDA in toluene cosolvent at $-78^{\circ} \mathrm{C}$. The curve depicts an unweighted least-squares fit to $k_{\mathrm{obsd}}=a[n-\mathrm{BuLi}]^{b}\left(a=1.79 \pm 0.08 \times 10^{-3}, b=0.96 \pm\right.$ $0.06)$. Alternatively, fit to $k_{\mathrm{obsd}}=a[n-\mathrm{BuLi}]^{b}+k^{\prime}[n-\mathrm{BuLi}]^{1 / 2}\left(a=1.75 \pm 0.08 \times 10^{-3}, b=\right.$ $0.97 \pm 0.06$ ), where $k^{\prime}=3.6 \times 10^{-5}$, the $b$ term from Section XIII with $4.0 \mathrm{M} \mathrm{Et}_{2} \mathrm{O}$.

XVII. Table of data for plot in Section XVI.

$\begin{array}{lllll}{[n-\mathrm{BuLi}](\mathrm{M})} & k_{\mathrm{obsd}} 1\left(\mathrm{~s}^{-1}\right) & k_{\mathrm{obsd}} 2\left(\mathrm{~s}^{-1}\right) & k_{\mathrm{obsd}} 3\left(\mathrm{~s}^{-1}\right) & k_{\mathrm{obsd}}(\mathrm{av})\left(\mathrm{s}^{-1}\right) \\ 0.05 & 0.000144 \pm 2 \mathrm{E}-6 & 0.000168 \pm 2 \mathrm{E}-6 & \\ 0.10 & 0.000236 \pm 2 \mathrm{E}-6 & 0.000245 \pm 3 \mathrm{E}-6 & 0.00016 \pm 2 \mathrm{E}-5 \\ 0.20 & 0.000353 \pm 6 \mathrm{E}-6 & 0.000467 \pm 6 \mathrm{E}-6 & 0.000240 \pm 6 \mathrm{E}-6 \\ 0.25 & 0.000436 \pm 6 \mathrm{E}-6 & 0.000392 \pm 6 \mathrm{E}-6 & 0.00041 \pm 8 \mathrm{E}-6 \\ 0.35 & 0.00062 \pm 2 \mathrm{E}-5 & 0.00056 \pm 1 \mathrm{E}-5 & 0.00041 \pm 3 \mathrm{E}-5 \\ 0.40 & 0.00077 \pm 2 \mathrm{E}-5 & 0.000747 \pm 2 \mathrm{E}-6 & & 0.00059 \pm 4 \mathrm{E}-5 \\ 0.50 & 0.00092 \pm 3 \mathrm{E}-5 & 0.00099 \pm 4 \mathrm{E}-5 & & 0.00076 \pm 2 \mathrm{E}-5 \\ 0.60 & 0.00106 \pm 5 \mathrm{E}-5 & 0.00113 \pm 7 \mathrm{E}-5 & 0.00103 \pm 4 \mathrm{E}-5 & 0.00107 \pm 5 \mathrm{E}-5 \\ 0.70 & 0.00139 \pm 5 \mathrm{E}-5 & 0.00129 \pm 4 \mathrm{E}-5 & 0.00120 \pm 4 \mathrm{E}-5 & 0.00129 \pm 9 \mathrm{E}-5\end{array}$




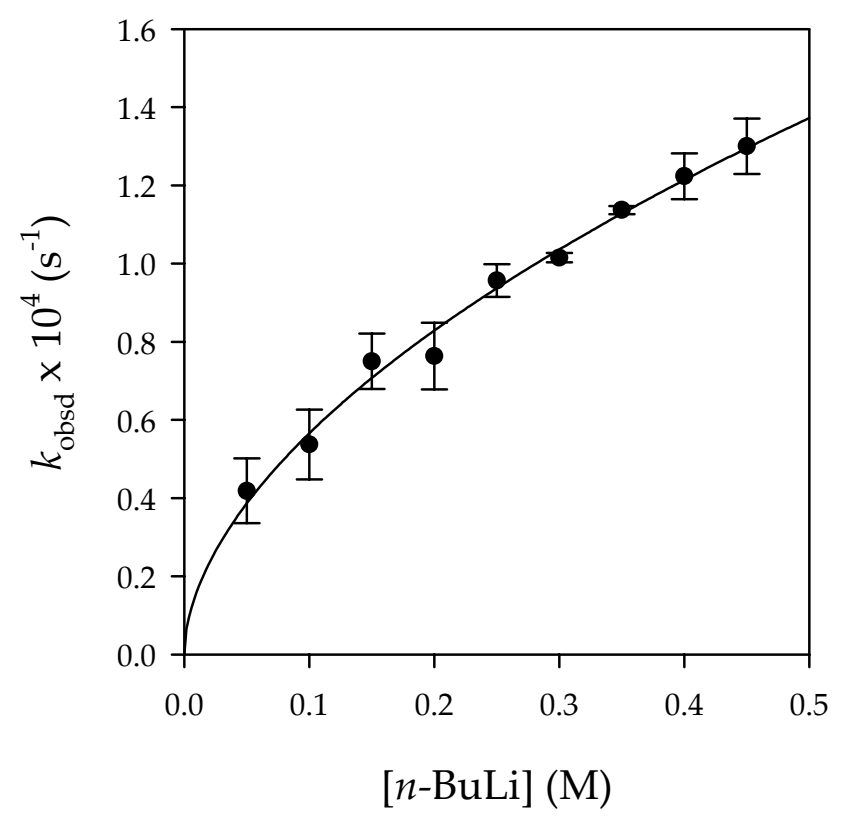

XVIII. Plot of $k_{\text {obsd }}$ vs [ $n$-BuLi] for the 1,2-addition of $n$ - $\operatorname{BuLi}(0.10 \mathrm{M})$ to imine $\mathbf{1}$ (0.007 $\mathrm{M})$ in $6.0 \mathrm{M} \mathrm{Et}_{2} \mathrm{O}$ and $0.90 \mathrm{M}$ TMEDA in toluene cosolvent at $-78^{\circ} \mathrm{C}$. The curve depicts an unweighted least-squares fit to $k_{\mathrm{obsd}}=a[n-\mathrm{BuLi}]^{b}\left(a=2.01 \pm 0.07 \times 10^{-4}, b=0.55 \pm\right.$ $0.03)$.

XIX. Table of data for plot in Section XVIII.

$\begin{array}{lllll}{[n-\mathrm{BuLi}](\mathrm{M})} & k_{\text {obsd }} 1\left(\mathrm{~s}^{-1}\right) & k_{\text {obsd }} 2\left(\mathrm{~s}^{-1}\right) & k_{\text {obsd }} 3\left(\mathrm{~s}^{-1}\right) & k_{\text {obsd }}(\mathrm{av})\left(\mathrm{s}^{-1}\right) \\ & & & & \\ 0.05 & 0.000036 \pm 1 \mathrm{E}-6 & 0.0000477 \pm 8 \mathrm{E}-7 & & 0.000042 \pm 8 \mathrm{E}-6 \\ 0.10 & 0.0000474 \pm 9 \mathrm{E}-7 & 0.000060 \pm 3 \mathrm{E}-6 & & 0.000054 \pm 9 \mathrm{E}-6 \\ 0.15 & 0.00008 \pm 2 \mathrm{E}-5 & 0.00007 \pm 2 \mathrm{E}-5 & & 0.000075 \pm 7 \mathrm{E}-6 \\ 0.20 & 0.000068 \pm 2 \mathrm{E}-6 & 0.000076 \pm 1 \mathrm{E}-6 & 0.000085 \pm 2 \mathrm{E}-6 & 0.000076 \pm 8 \mathrm{E}-6 \\ 0.25 & 0.000099 \pm 1 \mathrm{E}-6 & 0.000097 \pm 2 \mathrm{E}-6 & 0.000091 \pm 2 \mathrm{E}-6 & 0.000096 \pm 4 \mathrm{E}-6 \\ 0.30 & 0.000093 \pm 2 \mathrm{E}-6 & 0.000110 \pm 1 \mathrm{E}-6 & & 0.00010 \pm 1 \mathrm{E}-5 \\ 0.35 & 0.000114 \pm 1 \mathrm{E}-6 & 0.000103 \pm 3 \mathrm{E}-6 & 0.000124 \pm 2 \mathrm{E}-6 & 0.00011 \pm 1 \mathrm{E}-5 \\ 0.40 & 0.000120 \pm 3 \mathrm{E}-6 & 0.000118 \pm 2 \mathrm{E}-6 & 0.000129 \pm 1 \mathrm{E}-6 & 0.000122 \pm 6 \mathrm{E}-6 \\ 0.45 & 0.00125 \pm 4 \mathrm{E}-5 & 0.000135 \pm 3 \mathrm{E}-6 & & 0.00013 \pm 7 \mathrm{E}-6\end{array}$




\section{Experimental Section}

I. General procedures.

Reagents and solvents. Toluene, cumene, hexane, and $\mathrm{Et}_{2} \mathrm{O}$ were vacuum transferred through the vacuum line from degassed blue or purple stills containing sodium benzophenone ketyl. The solvent was collected in a pear-shaped flask in a dry ice/acetone bath. Air- and moisture- sensitive materials were manipulated using vacuum line and syringe techniques. TMEDA was purchased from Aldrich and purified by recrystallizing TMEDA $\bullet 2 \mathrm{HCl}$ two times from $\mathrm{MeOH} / \mathrm{H}_{2} \mathrm{O}$. The ammonium salts were mixed in the solid phase with solid $\mathrm{KOH}$. The resulting liquid was decanted and distilled. The wet liquid was further sequentially distilled from $\mathrm{CaH}_{2} / \mathrm{KH}, \mathrm{Na} /$ bezophenone before use (Rennels, R. A.; Maliakal, A. J.; Collum, D. B. J. Am. Chem. Soc. 1998, 120, 421, supporting information).

Purification of n-BuLi. A $100 \mathrm{~mL}$ of Aldrich-purchased 1.6 M n-BuLi in hexane was transferred with a canuula to a $200 \mathrm{~mL}$ Schlenk flask fitted with a stir bar. The solution was vacuum concentrated to about half the volume and titrated with diphenylacetic acid in THF (Kofron, W. G.; Baclawski, L. M. J. Org. Chem. 1976, 41, 1879) to ensure that the concentration of the resulting solution is between 3.0 and $3.5 \mathrm{M}$. The solution was then shocked in liquid nitrogen for 20-30 sec and then placed in a $-94{ }^{\circ} \mathrm{C}$ freezer for $\geq 72 \mathrm{~h}$. The resulting suspension was placed in a $-78{ }^{\circ} \mathrm{C}$ dry ice/acetone bath. The upper solvent was removed via cannula to another argon filled Schlenk flask, diluted with hexane and quenched with methanol before disposal. The crystals that remained were again recrystallized from a 3.0-3.5 $\mathrm{M}$ hexane solution. The resulting crystals were allowed to melt at room temperature and were diluted with freshly distilled hexane to the desired molarity (typically 2.5-3.5 M) as measured by repeated titration. This solution was then used as described below.

IR spectroscopic analyses. IR spectra were recorded using a ReactIR 1000 from ASI Applied System fitted with a 30-bounce silicon-tipped (SiComp) probe. A representative reaction was carried out as follows: The IR probe was inserted through a nylon adapter and FETFE O-ring seal (Ace Glass) into an oven-dried, cylindrical flask 
fitted with a magnetic stir bar and T-joint. The T-joint was capped by a septum for injections and an argon line. Following evacuation under full vacuum and flushing with argon, the flask was charged with a solution of $6.0 \mathrm{M} \mathrm{Et}_{2} \mathrm{O}(6.30 \mathrm{~mL}), 1.0 \mathrm{M}$ TMEDA $(1.51 \mathrm{~mL})$ in $1.77 \mathrm{~mL}$ toluene, and cooled in a $-78{ }^{\circ} \mathrm{C}$ dry ice/acetone bath. After $25 \mathrm{~min}$ of equilibration, $0.1 \mathrm{M}(0.382 \mathrm{~mL})$ of titrated $n$-BuLi was added to the cold flask. Further cooling for $5 \mathrm{~min}$, a background spectrum was recorded, and imine $\mathbf{1}$ ( $0.007 \mathrm{M}$ in hexane) was added with stirring. IR spectra were recorded over the course of the reaction. The rates of 1,2-addition of $n$-BuLi to imine 1 were monitored by following the loss of imine $\left(1667 \mathrm{~cm}^{-1}\right)$.

The disappearance of the imine was fit to the equation $[\mathrm{A}] /\left[\mathrm{A}_{0}\right]=\mathrm{a}^{*} \mathrm{e}^{-k_{\text {obsa }} \mathrm{t}}+\mathrm{c}$. Where $[\mathrm{A}]$ is the concentration of imine at time " $\mathrm{t}$ " and $\left[\mathrm{A}_{0}\right]$ is the concentration of imine at $t=0$. Term "c" is a measure of the amount of imine remaining at $t=\infty$, which is typically a positive number at $\leq 5 \%$ of $\left[\mathrm{A}_{0}\right]$

\section{Preparation of imine 1.}

Imine 1 (Betz, J.; Heuschmann, M. Tetrahedron Lett. 1995, 36, 4043) was prepared by condensation of $5.0 \mathrm{~mL}(0.046 \mathrm{~mol})$ pivalaldehyde with $12.0 \mathrm{~mL}(0.092 \mathrm{~mol})$ of (S)-1-methoxy-2-propanamine in the presence of $4 \AA$ molecular sieves. The reaction was finished after stirring at room temperature for $5 \mathrm{~h}$. Imine 1 was then distilled from the reaction mixture (5.42g, 75\% yield). ${ }^{1} \mathrm{H}$ NMR (500 MHz, $\left.\mathrm{CDCl}_{3}\right): \delta 7.39(1 \mathrm{H}, \mathrm{s})$, $3.21(2 \mathrm{H}, \mathrm{m}), 3.17(3 \mathrm{H}, \mathrm{s}), 3.13(1 \mathrm{H}, \mathrm{m}), 0.96(3 \mathrm{H}, \mathrm{d}, J=6.0 \mathrm{~Hz}), 0.91(9 \mathrm{H}, \mathrm{s}) .{ }^{13} \mathrm{C} \mathrm{NMR}$ $\left(125 \mathrm{MHz}, \mathrm{CDCl}_{3}\right): \delta 171.7,77.6,65.6,59.1,36.0,27.2,19.1$. 

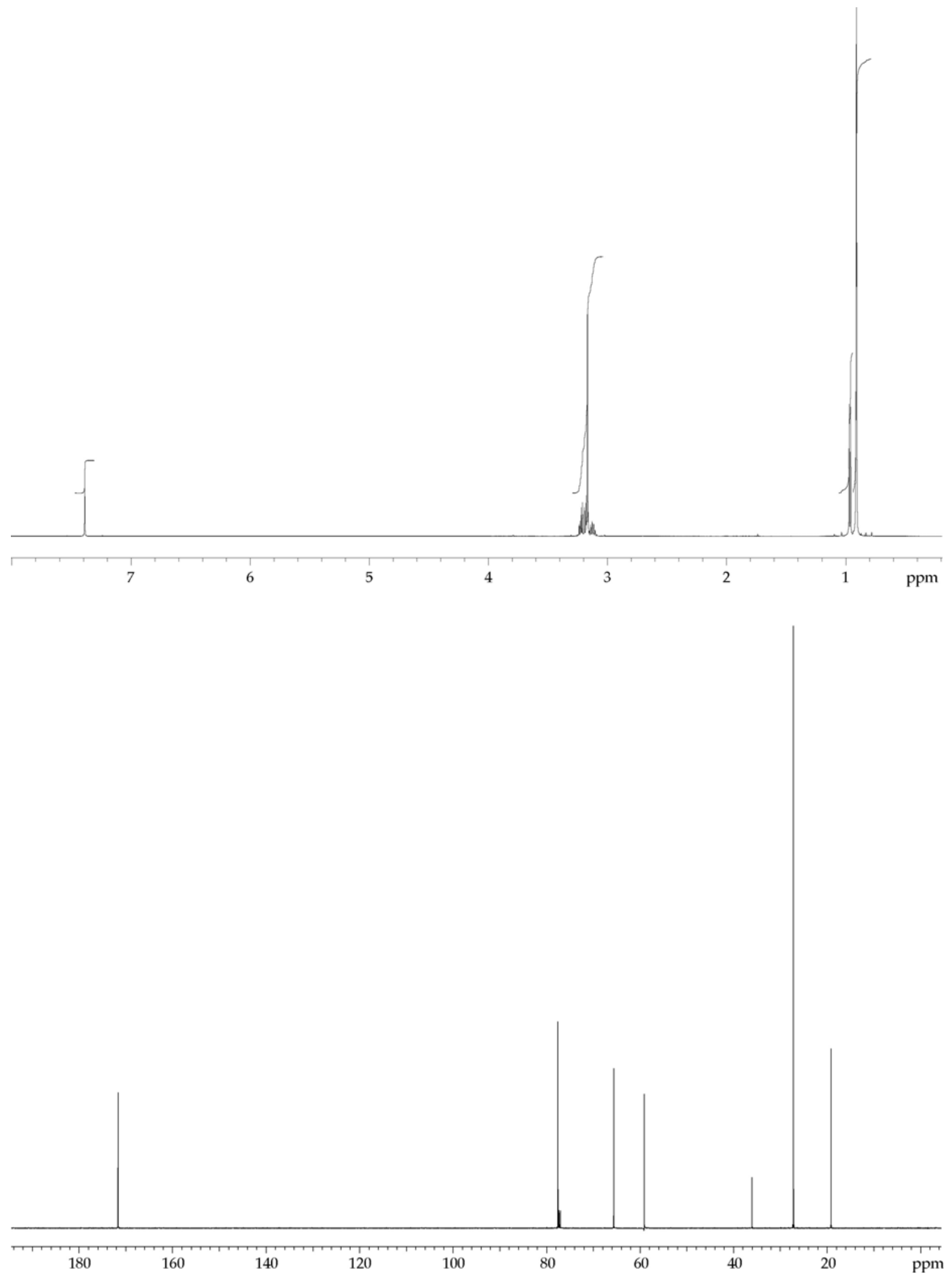